\title{
The Design and Evaluation of Personalised Ambient Mental Health Monitors
}

\author{
J.M. Blum and E.H. Magill \\ Department of Computing Science and Mathematics, University of Stirling, Stirling, UK \\ $\{\mathrm{jmb}, \mathrm{ehm}\} @$ cs.stir.ac.uk
}

\begin{abstract}
Mobile and environmental sensing technology can be used to assess human behaviour and mental health trajectories outside of laboratories and in ecologically-relevant settings. To achieve maximum benefit, the set of equipment and the monitoring patterns must be personalised to respect individual needs and fit into individual lifestyles.

We have developed a sensor network infrastructure for mobile phones and homecare using a rule-oriented programming architecture to monitor the activity signatures of people with Bipolar Disorder (BD). We believe that the use of this rule-based paradigm within the network for a mental health setting to be a contribution of this work.

We are evaluating the effectiveness of the technology in an ongoing technical trial with control participants as a precursor to studying the effectiveness of the system for use with people with BD. In this paper, we report the design and development of the monitoring system along with preliminary findings from the technical trial of the system, and discuss future developments.
\end{abstract}

\section{INTRODUCTION}

The last decade has seen the emergence of mobile and environmental sensor technologies used to monitor health concerns at home and in ambulatory settings. These technologies have been applied to mental health care under the auspices of ambulatory assessment [9]. Ambulatory assessment techniques record ecologically relevant data in real-time by monitoring subjects in-situ rather than in clinical settings. These techniques have shown advantages over traditional retrospective recall-based methods including the avoidance of biased recollection data, repeated assessment of variables of interest, and multi-modal context-rich data provision [1].

Many with BD endeavour to monitor their mental states for early warning signs of behavioural activity changes that alert them to impending manic or depressive episodes. Often such self-help includes the use of electronic or paper diaries to record mood [8]. These mood diaries allow BD sufferers to keep subjective records of their health.

The application of mobile and sensor network technologies to this type of monitoring brings with it novel development challenges related to system personalisation. Owing to interindividual differences in disease manifestation and differences within individuals over time, mental health care requires a higher level of system personalisation and technological integration than is typical for other types of sensor networks. The types of sensors used, and their patterns of usage, must match transient subject states and be acceptable to the subjects. We reviewed state-of-the-art work on sensor networks for psychiatric health monitoring in [2]. We found that many projects had been proposed for general medical care or homecare but, to the best of our knowledge, we are the first to attempt to obtain activity signatures from the mentally ill using a network of environmental and worn sensors. We also found that the existing projects had not shown how they respond to sparse datasets or integrate sensor data with self-monitoring reports.

The contribution of this work is the introduction, within a mental health setting, of a rule-based approach embedded within the network. This permits dynamic and straightforward personalisation of network behaviour. It also supports additional equipment as and when it becomes available.

To provide a more flexible approach to ambulatory assessment in the Personalised Ambient Monitoring (PAM) project, we monitor the activity signatures of individuals with BD. We employ an infrastructure (PAM-I) composed of offthe-shelf and custom built wireless sensors, and a rule-oriented programming architecture (PAM-A) to collect, process, and store data that can be related to models of care. We aim to augment existing self-help strategies with objective information from the sensors. The goal is to process the data within the network close to the sensors. In addition the network components will detect patient activity signatures, and combine this information with electronic mood diary data. We believe that by monitoring activity signatures in naturalistic environments, the system may detect changes in mental health and alert sufferers and their care providers to these changes early enough to maintain out-patient eurythmia.

We are assessing the PAM-I and PAM-A in trial stages. Firstly, we are assessing reliability and acceptability issues in a technical trial, to be followed by a feasibility study of the system using a small number of patients. Upon successful completion of the first two stages we will pursue a full clinical trial. Funding is currently in place for the first two stages.

The technical trial has begun and is taking place at four sites in the homes of project team members. Although this stage does not involve patients, it has been used by participants selected from the PAM project to exercise aspects of the subsequent patient trial, so ethical approval was obtained from the three universities within the project. In addition to sensor data collection, interviews were conducted to gather the views of those being monitored before and after each trial.

This paper reports on progress and preliminary findings from the first stage. We discuss the design and development of PAM-I and PAM-A along with preliminary findings from our technical trial of the system, and future developments. 


\section{SYSTEM DESIGN}

The PAM system is proposed for ambient mental health data collection and for reporting meaningful information to patients and care providers. The system consists of two parts. Firstly an infrastructure (PAM-I) composed of off-the-shelf and custom built wireless sensors, and secondly a programming architecture (PAM-A) that uses rules to control monitoring settings and stream data for processing. Figure 1 shows the relationship between PAM-I and PAM-A.

\section{A. PAM-I}

The Personalised Ambient Monitoring Infrastructure (PAMI) is a wireless network of wearable sensor nodes, mobile phones, a personal computer (PC) and environmental sensor nodes installed in subjects' homes. PAM-I uses the Bluetooth protocol to connect wearable nodes with mobile phones. Bluetooth is also used between phones and in-home PCs. Multiple communications standards are used by the environmental sensors and the PC including wireless IEEE $802.11 \mathrm{~b} / \mathrm{g}, \mathrm{X} 10$, and Bluetooth. The PC also requires an Internet connection for secure off site data storage.

Sensor nodes are composed of one or more sensors, computing capabilities, and communications devices. The nodes may be mobile and require battery power, or may be placed in a single location and be plugged into a wall socket. In many cases the computing capabilities are restricted to support small form factors and prolong battery life. The number of wearable devices is minimised to at most three (including mobile phone) for user acceptability reasons.

PAM-I currently supports two types of on-body nodes. Custom made wearable nodes include three-axis accelerometers, ambient light detectors, and ambient sound

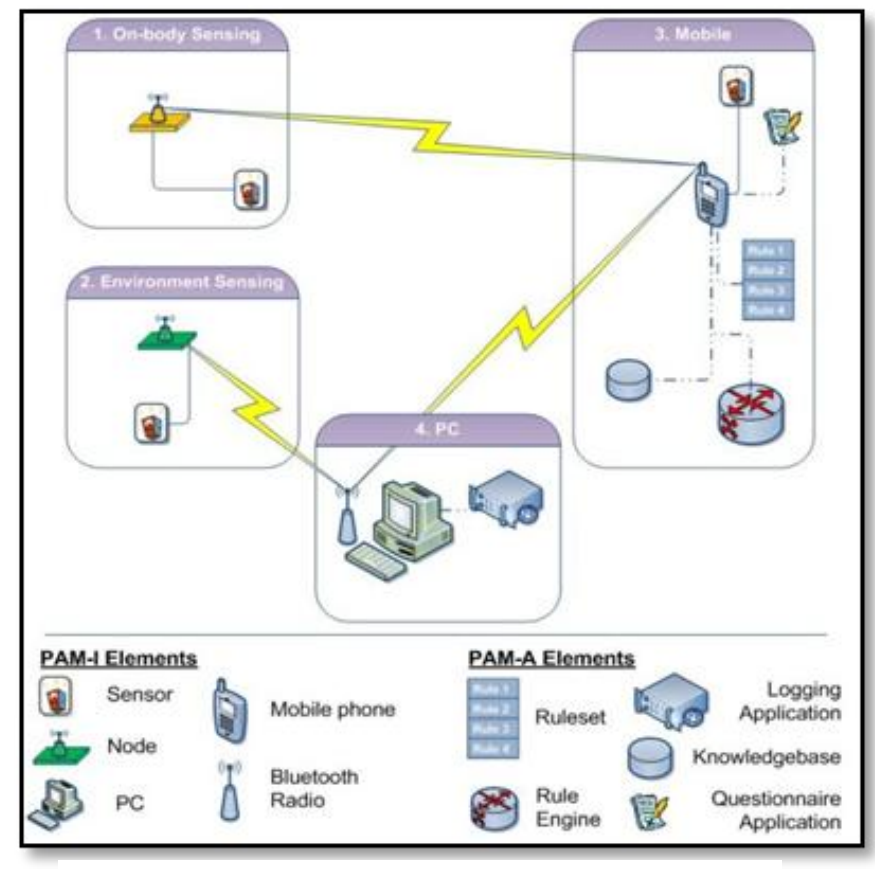

Figure 1. Graphical view combining PAM-I and PAM-A sensors. Off-the-shelf GPS transceivers are also supported. Each on-body device is the size of a matchbox. Wearable nodes can be worn on belts or strapped to arms. GPS units can be carried in pockets. Data is streamed from the sensors to the subjects' mobile phones. These are used to collect data from sensors and transmit data to PCs for storage and analysis. Mobile phones control sensor collection using rule-oriented applications. Additionally, momentary assessment of subject activities and moods can be collected through personalised onphone questionnaires.

Various environmental sensors are supported by PAM-I. These sensors are used to collect data about in-home subject activity. General home activity is monitored using light and sound level detectors, along with passive infrared sensors for monitoring how subjects are moving between rooms. Kitchen activity is monitored using a combination of micro-switches placed on kitchen cupboard doors and a wide-angle camera for monitoring many areas of interest (such as refrigerators and ovens) at once. Images are processed to extract activity data, and only activity data is stored to preserve subject privacy. Additionally, there are sensors for monitoring television usage and sleep patterns.

\section{B. PAM-A}

The Personalised Ambient Monitoring Architecture (PAMA) is composed of custom applications to handle inter-device network connections, control data streaming frequencies, record streamed data to persistent storage and transfer data offsite for long term storage and analysis.

PAM-A is supported on the mobile phone and PC. Applications are programmed using a mixture of Java for device control and Prolog for rule processing to personalise the system. Data streamed from devices are stored in XML documents conforming to the PAM sensor reading schema $\left(\mathrm{PSR}^{1}\right)$. Sensor readings from heterogeneous devices can be stored in a single PSR file. The format is intended to:

1. Allow readings from different devices to be interlaced throughout the file

2. Group readings into sets

3. Keep verbosity to a minimum in order to maximise batterypowered device lifetimes

Four custom PAM-A applications were written for mobile phones. PAM-Gateway can control data capture from the wearable units, PAM-Transfer performs mobile-to-PC data transmission, $P A M-Q$ is a momentary assessment questionnaire application, and PAM-Pause is a second questionnaire to record the reasons why the subject is pausing the monitoring system. The applications were programmed using Java ME for Symbian S60 3rd Ed.[3] phones and M-Prolog [4].

The PAM-Gateway application provides services to connect to wearable sensor devices and to access internal phone sensors, such as Bluetooth encounter monitoring. The set of sensors the gateway communicates with, and the data

\footnotetext{
${ }^{1}$ Normative and non-normative descriptions of the language and examples can be found online at http://www.cs.stir.ac.uk/ jmb/pam/readingXml/.
} 
streaming rates from the sensors, are controlled dynamically at run-time through rulesets. The application interfaces with a rule engine that is used to select appropriate settings on application startup, or upon device reconnection. PAMGateway provides a pause/resume monitoring service and handles device disconnection/reconnection gracefully. The application stores readings from multiple data sources to files ready for transfer to the PC using PAM-Transfer.

At the heart of PAM-Gateway is a device mediator that can load a ruleset into its rule-engine at run-time. It can connect to a set of sensor nodes, control their sensing characteristics and store readings from them. The mediator can also instantiate first-class "Action" objects in response to rule conclusions about the state of the mediator's knowledgebase. Device handler objects are instantiated at application start-up depending on the rules used to personalise the set of nodes for each user. The sensors may be on-phone sensors (such as GPS and accelerometer) or external nodes capable of Bluetooth communication. Rules contain handler names and configuration options such as data collection frequencies. Once instantiated, device handlers attempt to connect to their devices and forward control settings to them. Handlers maintian connections and respond to unexpected disconnections by automatically attempting to reconnect. Each handler registers a corresponding listener that listens for, and responds to, new data. Responses include recording the data or loading actions into the mediator as a result of a rule engine query.

Data is written for each sensor into the same PSR file corresponding to the PAM-Gateway session. Each reading belongs to a reading set describing the type of reading and the frequency that the reading was taken at. New reading sets are generated each time a device changes state (for instance if a device records data from alternating sensors). This provides information about the period the readings were taken along with the reading data, which supports the understanding of activity across sensors.

PAM-Q and PAM-Pause provide sets of questions and possible answers to select for each question. Questionnaires present themselves periodically to users. PAM-Q and PAMPause are personalisable. The questions and answer choices, along with settings controlling notification periods are stored in the knowledgebase and are loaded dynamically at run time.

Applications also run on the home PC to interface with the mobile phone, environmental sensors, and backup the data for long-term storage. A Java J2SE application runs on the PC for interfacing with PAM-Transfer as well as securely storing and backing up mobile and environmental data.

\section{TECHNICAL TRIAL}

We designed a technical trial of the PAM system to assess its reliability and acceptability. The technical trial was composed of two semi-structured interviews, and a raw data collection period. On-body data was stored on technical trial mobile phones and transferred to technical trial PCs installed in the participants' homes. The PCs also received environmental sensor readings. The data were encrypted and backed-up offsite.

We selected four study participants from the PAM project team for the technical trial. To be included in the technical trial the participants provided ongoing informed consent and were free of serious health conditions that could have been aggravated by monitoring. Participants could have withdrawn from any part of the trial at any point in time. One participant completed the technical trial at the time of writing and the three others were still in the data monitoring phase.

Participants were briefed about the data collection and processing prior to beginning the technical trial, and also during a training session that followed device installation. They were shown how to record their answers to the questionnaires, and were familiarised with the importance of using PAM-Pause to distinguish deliberate pausing from missing data values arising from faults in the system.

\section{A. Interview Procedures}

Qualitative semi-structured interviews were designed to capture detailed points of view regarding the PAM project, its technology and monitoring in general. The entrance interview was focused on cultural factors as well as system acceptability and compliance issues. The exit interview was designed to review participation and to elicit thoughts and feelings about system acceptability, compliance and technological reliability.

\section{B. Data Collection}

PAM technical trial data collection involved sensors being worn by the participants and placed in their homes. Customised mobile phones were provided to coordinate sensor communication and collect information about the participants' activities of daily life. The participants were asked to record their activities using a questionnaire application on the mobile phone. Continuous, discreet time-based sampling strategies were used to cover the variety of heterogeneous devices.

On-body device sampling rates were chosen to balance signal-processing concerns with the limitations of the wearable node processor and mobile phone memory constraints. The wearable nodes were not able to transmit accelerometer data and microphone data at the same time. Instead, rules programmed them to alternate transmiting accelerometer and light meter data with microphone data. Transmissions alternated between five minutes of accelerometer data at 20 recordings per second along with the light meter once per second, and then one minute of microphone recordings at 10 samples per second. The ordering and time values were set in the ruleset at these rates for data analysis purposes. Raw data was streamed continuously from the wearable to the trial mobile phone where they were stored and forwarded to the subjects' trial PCs. The mobile phone also recorded data streamed from the external GPS node once every four seconds.

The mobile phone was set to display PAM-Q application notifications every hour. This sampling rate was determined in consideration of advice on time-based study design reported in 
[5]. Our experiments showed that two questions in the questionnaire could be answered in less than 10 seconds if the application was already opened and in less than 20 seconds if the application needed to be launched. A rule set notifications to fire every hour in order to minimise self-report burden by fitting in to the average schedule boundaries of the participants. Notifications could have been ignored by the participants, and they could have entered questionnaire data at any time regardless of notification timing. Each sample was timestampped on questionnaire response entry.

Two questions were available for answer on the mobile phone. The response-set (happy, interested, anxious, angry, sad, ashamed, disgusted, other emotion, no emotion) for the question "How Are You Feeling?" was added to the ruleset derived from the basic emotions reported in [7]. The responses to the second question "What Are You Doing?" were personalised for each participant to correspond to the most likely high-level activities (such as commuting or working) and low-level activities (such as walking and sitting) performed. Multiple responses were selectable for each question.

Most of the environmental sensors performed continuous monitoring. The passive infrared sensors (PIR), microswitches, bed sensor and remote control usage sensor transmitted data on event-triggers. The mininmum time between PIR sample transmission was between 6 and 10 seconds. The camera transmitted one picture of the kitchen every 10 seconds to the in-home PC. The images were immediately processed to look for activities in areas of interest and only the activity data was stored. Ambient light and sound level samples were continuously streamed to the in-home PC over Bluetooth.

\section{TECHNICAL TRIAL PRELIMINARY FindingS}

All four participants have begun the technical trial and one has completed it, however data has been recorded for all the participants. All the participants were interviewed before the sensors were installed in their homes. The participant that completed the trial was monitored for a month and was also interviewed after the completion of the monitoring phase.

The entrance interviews revealed some similarities in the participants' thoughts and feelings about project characteristics. All four participants were male and had a high degree of exposure to the trial equipment and technical trial protocols prior to beginning the technical trial. They all lived with at least one other housemate and sought permission of their housemates prior to installing the equipment. The participants had no or little prior experience of being monitored outside of the PAM project. They all expected to have an initial period of self-consciousness at the beginning of the trial that they presumed would lessen, but they didn't expect the monitoring to affect their general daily patterns of activity.

The entrance interviews also revealed distinct differences between the participants. For three of the participants, their feelings about the degree of monitoring in society ranged from generally negative, but acknowledging value for medical purposes, to wholy positive (no response was recorded for the fourth participant). Their views about how much time per day they thought was appropriate to spend interacting with the technical trial devices ranged from one minute per day or less up to 20 minutes. Three of the participants had some form factor issues with either the wearable unit or some of the environmental sensors. Interesting characteristics were revealed by comparing the collected data with the entrance and exit interview responses from the participant that completed the trial. His participation highlighted reactivity, compliance and reliability issues to do with the technology and study design.

The subject's entrance interview revealed he had not been monitored before and was mildly concerned about exposing his life patterns to the other researchers. He required assurance that the data was anonymised. In particular the household was concerned with camera data. He related in his exit interview that their concerns were alleviated when shown processed camera images, as these contained no identifiable information. The subject consented to the installation of all of the equipment except for the bed sensor. He allowed all of the chosen devices to record data through the duration of the technical trial and never considered ending his participation early. No installation concerns were reported by the subject during the entrance interview other than about making sure that the sensors would not mark the paint on his walls. However, during his exit interview, the participant expressed concern regarding the installation procedures. He described a sense of loss of control owing to having three people taking part in the installation and a feeling that the installation took too long.

The subject and housemates adapted quickly to most of the environmental sensors, but reacted in an unexpected way to the camera. The subject expected that the household would be selfconscious for the first couple of days but would not alter their behaviours because of being monitored. However, the subject reported in the exit interview that his household forgot about most of the monitoring equipment quite quickly except for the camera (because of its distracting LED).

The subject found the system quite obtrusive and his compliance was affected by reliability issues. He reported poor mobile phone battery life, intermittent device disconnection problems, and form factor issues related to carrying three devices. He found the devices burdensome and worried about dropping them or leaving them behind. The wearable node irritated the participant because of its positioning and dropped communication connections.

The participant reported positive feelings towards the mobile phone's form factor and screen quality, but he found the mobile phone questionnaire and transfer applications burdensome, and reported a fairly low compliance rate. $\mathrm{He}$ reported in the entrance interview that mobile application notifications every hour would be useful and an appropriate time setting, however he would have preferred the notifications to be triggered by changes of activity rather than timeout- 


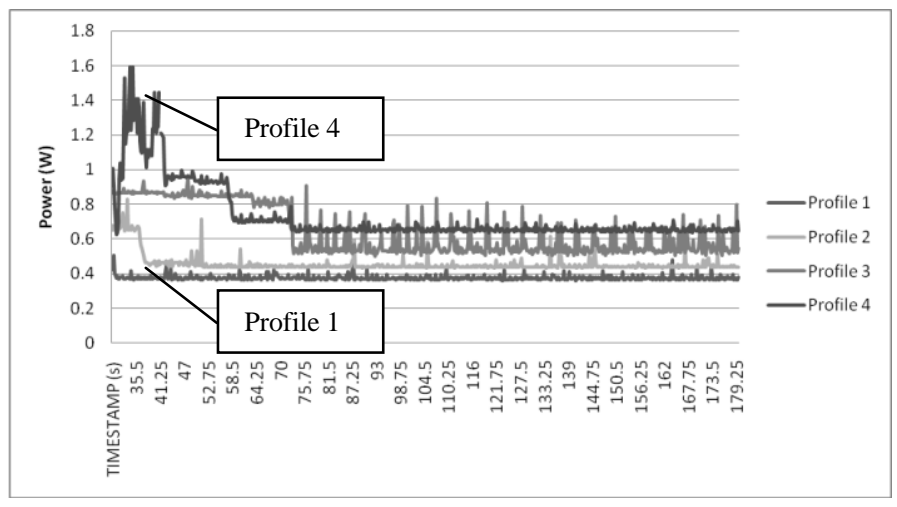

Figure 2. Power profiles for mobile phone - wearable node connection over Bluetooth.

based. In the entrance interview he believed that mobile questionnaire application interaction would be fine if it took less than one minute to complete per day. However, he reported that even though answering questionnaires was a quick process it was still overly intrusive and his compliance was low. He also reported that he missed many on-screen notifications and preferred audio-based notifications.

The PAM team investigated mobile battery life performance. We found that continuous data streaming over Bluetooth reduced the battery life by about $15 \%$ and file writing reduced it a further $25 \%$. We examined the mobile phone's battery life using the Nokia Energy Profiler version 1.2 with different sensor configurations. We expected that Bluetooth data streaming would be the largest power drain. Instead we found that the internal GPS unit (which we have subsequently disabled) and file writing to flash memory were much bigger power draws. Figure 2 shows consumption profiles. It compares tests starting 30 seconds into the recordings and running for three minutes. Profile 1 shows the mobile phone disconnected from the wearable node and not running any user applications. Its expected battery life was 9 hours at $0.41 \mathrm{~W}$ on average. Profile 2 shows data streaming from the wearable node to the mobile phone over Bluetooth, but without any onphone data storage. Its expected battery life was 7 and a half hours at an average watt consumption of 0.48. Profile 3 is the same configuration as profile 2 except that the data was being written to the phone's flash memory card. There was a lot more activity for profile 3 than there was for profile 2. Profile 3's expected battery life was 5 and a half hours at an average watt consumption of 0.67 . Profile 4 shows the power consumption for accessing the mobile phone's internal GPS. It had the highest impact on battery life with an average watt consumption of 0.68 and expected battery life of 5 hours.

\section{DISCUSSION \& FUTURE WORK}

We have begun the evaluation of the effectiveness of the PAM system. The technical trial highlighted reliability and acceptability issues regarding on-body and in-home technology including mobile phone battery life, on-body gateway disconnection, on-body device form factor issues, and environmental sensor reliability issues. The PAM team has revised the software driving the components to improve the system performance. The improved system is being evaluated by the three remainder participants of the technical trial.

Upon trial completion the data collected will be analysed to evaluate the effectiveness of the system in capturing behavioural patterns. Sensor data will be interpreted and compared to the user-recorded activities. Comparing the answers from the interviews, along with reviewing the data may contextualise data captured during the technical trial and help us improve system acceptability and operation during the feasibility study. A key motivation here is the feasibility of such technology for effective mental health monitoring.

Crucially PAM has also begun preparations for the feasibility study which includes BD patients. Based on our technical trial findiings we are revising our installation and training procedures, processing data in-network to improve onbody battery life, and provide backend support for network monitoring, rule provision, and data analysis.

\section{ACKNOWLEDGMENT}

We wish to thank the Engineering and Physical Sciences Research Council (EPSRC) for funding the PAM project and the Universities of Nottingham, Southampton and Stirling for their support. We also thank our colleagues on the PAM project for their participation in the technical trial and its design. In particular colleagues from the University of Nottingham for their work on wearable and environmental sensors, and the University of Southampton for their work on wireless cameras, activity detection algorithms, and help with the design of the interview protocol.

\section{REFERENCES}

[1] U.W. Ebner-Priemer, and T.J. Trull, "Ambulatory Assessment - An Innovative and Promising Approach for Clinical Psychology" European Psychologist, vol. 14, pp. 109-119, 2009.

[2] J Blum and E.H. Magill, "M-Psychiatry: Sensor Networks for Psychiatric Health Monitoring," PGNET 2008: The 9th Annual Postgraduate Symposium on The Convergence of Telecommunications, Networking and Broadcasting. Liverpool, June, 2008.

[3] "S60 platform overview", http://www.forum.nokia.com/main/platforms/s60/, accessed August, 2009.

[4] "mProlog: Lightweight PROLOG Engine" website, http://www.cs.uu.nl/3apl-m/mprolog.html, last accessed August, 2009.

[5] U.W. Ebner-Priemer and G. Sawitzki, "Ambulatory Assessment of Affective Instability in Borderline Personality Disorder - The Effect of the Sampling Frequency," vol. 23(4), pp. 238-247, 2007.

[6] A. Bryman, "A. Social research methods: Chapter 15 Interviewing in qualitative research," Oxford University Press New York, 2004.

[7] U.W. Ebner-Priemer et al., "Psychophysiological ambulatory assessment of affective dysregulation in borderline personality disorder," Psychiatry Research, vol. 150(3), pp. 265-275, 2007.

[8] S. Lane, N. Heddle, E. Arnold, and I. Walker, "A review of randomized controlled trials comparing the effectiveness of hand held computers with paper methods for data collection," BMC Medical Informatics and Decision Making, vol. 6, May 2006.

[9] J. Fahrenberg, "Assessment in daily life. A Review of Computer-assisted Methodologies and Applications in Psychology and Psychophysiology (years 2000-2005)." website, http://www.ambulatory-assessment.org, last accessed September, 2009. 